\title{
Signal detection in a paired-associate learning task ${ }^{1}$
}

\section{R. H. HICKSON, University of Arkansas, Fayetteville, Ark. 72701}

A paired-associate learning task was modified to produce a yes-no signal detection task. The correct response syllable was presented with the stimulus syllable for 10,25, 50, or $75 \%$ of trials with an incorrect, or "noise," member on remaining trials. This produced differences in rate of identification of correct signals as well as differences in signal sensitivity, $d^{\prime}$, and decision criteria.

The theory of signal detectability (TSD) (Egan \& Clarke, 1966; Green \& Swets, 1966; Swets, Tanner, \& Birdsall, 1961) has been applied by Parks (1966) and by Murdock (1965, 1966) to problems of short-term recognition-memory. In these studies, CVC trigrams or pairs of common English words were presented once in a list and Ss were then given a recognition test in which they were required to distinguish stimuli which had been previously presented from those which had not. Both investigators generally endorsed the applicability of TSD to the study of short-term memory. Murdock (1965) concluded that short-term memory seems like the continuous process postulated by TSD with the individual learner capable of varying his decision criterion over a wide range. The experiment described below is to test the applicability of TSD methods to changes in choice responses in learning paired-associate nonsense syllables.

In typical paired-associate learning, $S$ is required to produce a response which he thinks correctly belongs with the stimulus syllable which is being presented. A modification of this procedure is to present a response syllable with the stimulus syllable and require $S$ to say whether it is the correct response syllable. The correct S-R pair is then shown so that he is informed of the correctness of his decision. A difference between this forcedchoice task and the traditional signal detection task is that at the beginning of learning all the stimuli are "noise." Unlike a 1000 cycle tone occurring at different intensities in a background of noise, the correct response syllable, at the beginning of learning, has no greater tendency to evoke a response of "yes, I think this is a signal," than does an incorrect syllable. A learning experiment may be regarded, then, as a situation in which the tendency to recognize signals increases and the tendency to accept noise decreases. There is an increased probability that $S$ will accept the hypothesis that a signal is present when the observation which $S$ makes indicates that a signal is present, when he states that a signal is present, and when he is informed that his choice was correct.

The change in per cent of signals correctly identified is related to two central concepts in signal detection: (a) the strength of the signal relative to the strength of noise, and (b) the observer's response bias or decision criterion. The first of these is represented by the distance between the mean of the signal distribution and the mean of the noise distribution and is called $d^{\prime}$. Learning would result in a decrease in the overlap of these two distributions and a corresponding increase in $\mathrm{d}^{\prime}$. Secondly, response bias or decision criterion is represented by the cutoff on the observation continuum and summarizes S's tendency to identify a signal when one is presented (a hit) as a function of his tendency to accept noise as a correct signal (a false alarm). A high hit rate is achieved at the expense of a high false alarm rate and Ss vary in the balance which they wish to achieve between these two rates. Pollack \& Decker (1958) have described a method of confidence ratings which can be used to estimate this relationship between hit and false alarm rates. A modification of this method (Murdock, 1965) requires S on each trial to give an estimate, on a 5-point scale, of the confidence he has in the choice he has just made either to accept or reject the observation. These ratings are then used to construct receiver-operating-characteristic (ROC) curves which show hits as a function of false alarms and each S's performance can be described as a point on the curve.

The objective of this study is to show (a) that a priori probability of a correct signal affects rate of learning in this form of paired-associate nonsense syllable learning; (b) that a priori probability of a correct signal affects sensitivity to the signal (as described by $\mathrm{d}^{\prime}$ ) and response bias (as shown by the ROC curve); and (c) that sensitivity and response bias will change over learning trials.

\section{METHOD}

Subjects. Sixty students enrolled in undergraduate psychology courses at the University of Arkansas volunteered as Ss. Each S was randomly assigned to one of the four groups.

Apparatus. A Lafayette Instrument Company memory drum (model 303A) was modified to present a frame every $6 \mathrm{sec}$. The drum was placed on a narrow table in a $4 \times 4 \mathrm{ft}$ cubicle lined with soundproofing material. An aluminum panel containing two pushbuttons 4 in. apart and a ready light centered 4 in. above the pushbuttons was placed on the table to the left of the memory drum. These pushbuttons were used by $S$ to indicate either "Yes, I think this was a correct response member," or "No, I think this was an incorrect response member." The preferred finger of the left hand was used to make these responses. An aluminum panel containing five horizontally mounted pushbuttons 2 in. apart was placed on the table to the right of the memory drum. On each presentation of a syllable pair, $S$ pressed one of these buttons with the preferred finger of his right hand to indicate his confidence in the yes-no decision. Choice responses and confidence ratings were automatically recorded on an Esterline-Angus event recorder.

Stimulus materials. Ten pairs of low association nonsense syllables were selected from Hilgard's list (Stevens, 1951, p. 545) to conform to the rules for constructing lists. The list was arranged in 10 different random orders. In traditional paired-associate learning, the odd-numbered frames contain the stimulus member alone and the even-numbered frames contain the correct stimulus-response pair. In this study the stimulus member on odd-numbered frames was paired either with the correct response member or with an incorrect response member selected at random from the list of low association nonsense syllables. No incorrect syllable was used more than once during the experiment. The difference between the four experimental groups was in the frequency with which the stimulus syllable on odd-numbered frames was paired with the correct response syllable. These frequencies were $10,25,50$, and $75 \%$. The stimulus list for each group then consisted of 10 trials with 20 frames in each trial-a stimulus syllable in each odd-numbered frame paired with a correct or incorrect response syllable followed by an even-numbered frame which presented the correct stimulus-response pair.

Syllables were typed in capital letters. On odd-numbered frames the stimulus and response syllable were separated by a distance of 1 in. In even-numbered frames, when the correct pair was presented, the syllables were separated by a distance of 0.5 in.

For each $\mathrm{S}$, the list of nonsense syllables was preceded by five pairs of two digit numbers arranged in four different random orders. In odd-numbered frames the stimulus member was paired with a response member which was correct with a probability of $0.10,0.25,0.50$, or 0.75 . Correct pairs were presented in even-numbered frames. The four trials of five pairs of numbers were included simply to familiarize $S$ with the yes-no decision task and to show him that he could improve from trial to trial.

Procedure. Instructions were read to $\mathrm{S}$ before the warm-up trials with the

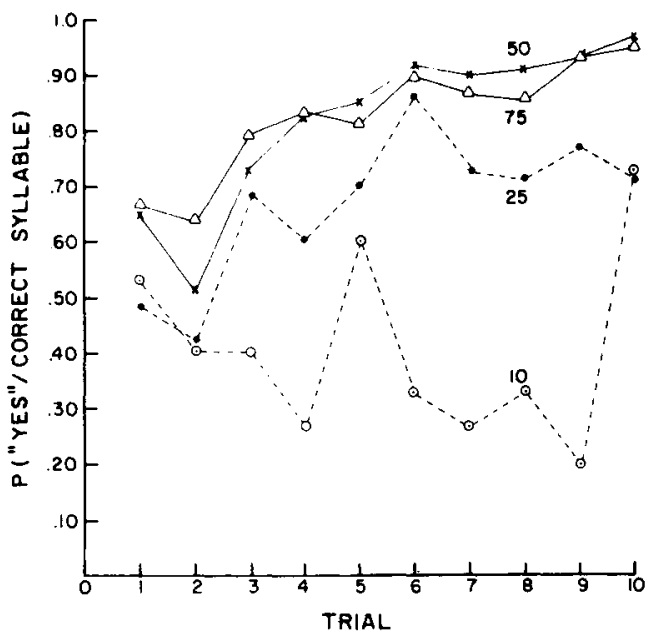

Fig. 1. Mean probability of saying yes given a correct response member for each trial and each group. 


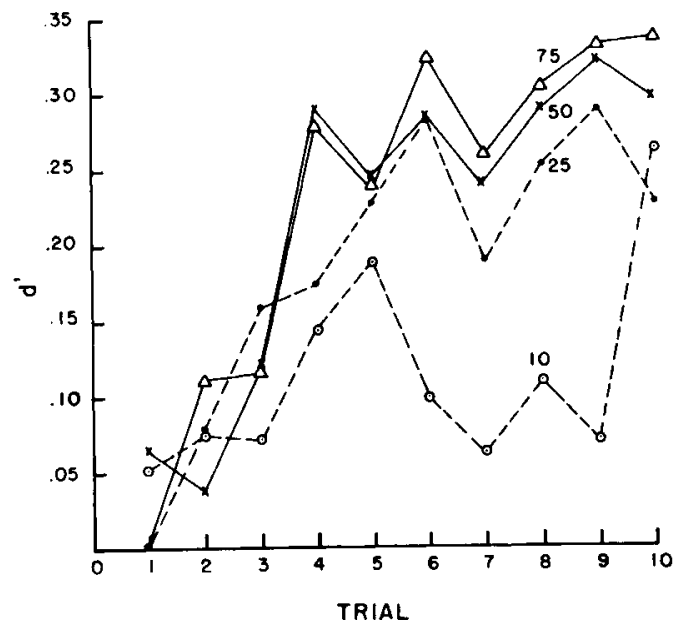

Fig. 2. Sensitivity to the signal, $d^{\prime}$, for each of the four groups as a function of trials.

number pairs. The instructions relevant to the decision task were "...try to improve your decisions on each series; try to make more and more correct choices as you see the series over and over and take each pair of syllables on its own merits. Simply ask yourself whether you think this is the correct pair or not." They were instructed to pronounce the syllables out loud and were told the per cent of pairs which would have the correct response member. RESULTS

Learning consists of both an increase in identification of signals and an increase in rejection of noise. When hits and correct rejections were combined and the mean for each group obtained for each of the 10 trials, an analysis of variance for trend showed no difference among the four groups alth ough learning occurred over trials. Separate analyses were then done for hits and for correct rejections. Figure 1 shows group mean probability of hits over the 10 trials. An analysis of variance of these data showed group differences $(F(3,50)$ $=14.77, p<.001)$, a difference in performance across trials $(F(9,504)=$ $7.61, \mathrm{p}<.001)$, and a trials by groups interaction $(\mathrm{F}(27,504)=2.98, \mathrm{p}<$ $.001)$. Each curve in Fig. 1 should eventually asymptote at 1.0 if Ss were to discriminate perfectly between correct and incorrect responses. A similar analysis of differences in the probability of saying no when an incorrect signal was presented (correct rejection) showed no differences among groups.

Following a me thod suggested by Parks (1966), a value of $d^{\prime}$ was calculated for each trial for each group by (a) obtaining the number of hits and false alarms for each trial; (b) expressing these as proportions of the number of signals and noise stimuli respectively per trial; (c) expressing these two proportions as

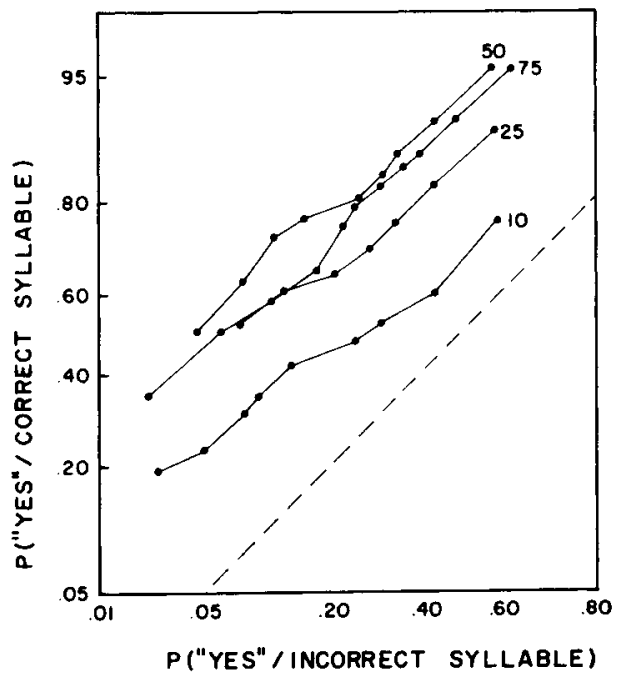

Fig. 3. ROC curve for each group with different per cent occurrence of correct response member. $z$-scores, i.e., finding the cutpoint on a normal curve above which the area equals the observed proportion; and (d) combining the two z-scores to obtain an estimate of $\mathrm{d}^{\prime}$. Figure 2 shows the estima tes of $\mathrm{d}^{\prime}$ for each group for each trial.

The observer's sensory capability for detecting the signal is expressed by $\mathrm{d}^{\prime}$ but it does not tell us what proportion of signals $S$ will correctly identify. Several observers may have the same ability to detect a signal but differ in the values which they place upon the four possible outcomes in this two-choice situation; viz., hits, misses, correct rejections, and false alarms. An ROC curve is a convenient summary, for a group of observers, of the balance which is achieved between hits and false alarms. Figure 3 shows an ROC curve for each of the groups. Murdock's (1965) method of confidence ratings was used to construct the curves shown in Fig. 3. When the proportion of hits and false alarms is plotted on double probability paper the result is a straight line if the assumption of normality of signal and noise is satisfied (Green \& Swets, 1966). Figure 3 shows that this assumption appears to be satisfied for each group. The broken line shows the major diagonal and represents chance performance or zero detectability.

\section{DISCUSSION}

The modified paired-associate learning task satisfactorily produced several response changes which could be described as learning. The total number of correct identifications of signals and noise increased over trials. The a priori probability of a signal, or correct -response member, the variable which distinguished the four groups, did not produce a difference in performance among the groups until hits were separated from correct rejections. Apparently a priori probability of signals and noise affects rate at which signals are identified but does not affect rate at which noise is rejected. The results leave unanswered the question of whether the differences among the four groups are due to differences in the frequency of presentation of the correct $S-R$ pair on odd-numbered trials or due to interference effects of new stimuli. The fact that there were no differences among groups in changes in probability of correct rejections over trials would suggest equal interference effects in all groups. However, Ekstrand et al (1966) have shown that in learning a verbaldiscrimination list, the cue for discrimination is the subjective difference in frequency of occurrence between the correct and incorrect alternative of a verbal-discrimination pair. In the present study not only proportion of correct stimuli differ among groups but also ratio of correct to incorrect pairs.

Figures 1 and 2 show a similarity between traditional learning curves and changes in sensitivity to the signal, $d^{\prime}$. This use of repeated random presentations of a paired-associate list extends Murdock's $(1965,1966)$ findings that short-term recognition-memory seems like the continuous process postulated by TSD. He also pointed out the capability of TSD for separating and describing the wide range of decision criteria employed by different Ss. Figure 3 summarizes both differences in signal sensitivity (distance from the major diagonal to each group curve) and variation within groups in decision criteria (the points along each ROC curve represent a ratio of hits to false alarms which can be adopted by a particular S). In general the data show that sensitivity to the signal can be separated from risk-taking propensities in a paired-associate learning task.

\section{REFERENCES}

EGAN, J. P., \& CLARKE, F. R. Psychophysics and signal detection. In J. B. Sidowski (Ed.), Experimental methods and instrumentation in psychology. New York: McGraw-Hill, 1966. Pp. 211-246.

EKSTRAND, B. R., WALLACE, W. P., \& UNDERWOOD, B. J. A frequency theory of verbal-discrimination learning. Psychological Review, 1966, 73, 566.

GREEN, D. M., \& SWETS, J. A. Signal detection theory and psychophysics. New York: John Wiley, 1966.

MURDOCK, B. B., Jr. Signal-detection theory and short-term memory. Journal of Experimental Psychology, 1965, 70, 443-447.

MURDOCK, B. B., Jr. The criterion problem in short-term memory. Journal of Experimental Psychology, 1966, 72, 317-324.

PARKS, T. E. Signal detectability theory of recognition-memory performance. Psychological Review, 1966, 73, 44-58.

POLLACK, I., \& DECKER, L. R. Confidence ratings, message reception, and the receiver operating characteristic. Journal of the Acoustical Society of America, 1958, 30, 286-292.

STEVENS (Ed.), Handbook of experimental psychology. New York: Wiley, 1951. Pp. 517.567.

SWETS, J. A., TANNER, W. P., Jr., \& BIRDSALL, R. G. Decision processes in perception. Psychological Review, 1961, 68, 301-340. NOTE

1. The author would like to thank Nancy Greenlee and Donna Hairston who assisted in collecting and tabulating the data. 